\title{
Neural and psychological maturation of decision-making in adolescence and young adulthood
}

Article

Published Version

Christakou, A., Gershman, S., Niv, Y., Simmons, A., Brammer, M. and Rubia, K. (2013) Neural and psychological maturation of decision-making in adolescence and young adulthood. Journal of Cognitive Neuroscience, 25 (11). pp. 1807-1823. ISSN 1530-8898 doi: https://doi.org/10.1162/jocn_a_00447 Available at https://centaur.reading.ac.uk/33026/

It is advisable to refer to the publisher's version if you intend to cite from the work. See Guidance on citing.

To link to this article DOI: http://dx.doi.org/10.1162/jocn_a_00447

Publisher: MIT Press

All outputs in CentAUR are protected by Intellectual Property Rights law, including copyright law. Copyright and IPR is retained by the creators or other copyright holders. Terms and conditions for use of this material are defined in the End User Agreement.

www.reading.ac.uk/centaur 
Central Archive at the University of Reading

Reading's research outputs online 


\title{
Neural and Psychological Maturation of Decision-making in Adolescence and Young Adulthood
}

\author{
Anastasia Christakou ${ }^{1,2}$, Samuel J. Gershman ${ }^{3}$, Yael Niv³, \\ Andrew Simmons ${ }^{2}$, Mick Brammer ${ }^{2}$, and Katya Rubia ${ }^{2}$
}

\begin{abstract}
We examined the maturation of decision-making from early adolescence to mid-adulthood using fMRI of a variant of the Iowa gambling task. We have previously shown that performance in this task relies on sensitivity to accumulating negative outcomes in ventromedial PFC and dorsolateral PFC. Here, we further formalize outcome evaluation (as driven by prediction errors $[\mathrm{PE}]$, using a reinforcement learning model) and examine its development. Task performance improved significantly during adolescence, stabilizing in adulthood. Performance relied on greater impact of negative compared with positive PEs, the relative impact of which matured from adolescence into adulthood. Adolescents also showed increased exploratory behavior, expressed as a propensity to shift responding between options independently of outcome quality, whereas
\end{abstract}

\section{INTRODUCTION}

Adolescence is characterized by disproportionately increased risk-taking and novelty-seeking (Casey, Getz, \& Galvan, 2008; Spear, 2000). Current neurodevelopmental models suggest that this profile depends on a hyperactive reward system readily activating consummatory behaviors (Galvan, 2010), although there is evidence that a hypoactive reward system may instead be promoting the pursuit of disproportionately large motivating stimuli (Bjork et al., 2004; Spear, 2000).

Substantial structural changes in the adolescent reward system affect dopaminergic (Teicher, Andersen, \& Hostetter, 1995) and frontostriatal mechanisms (Galvan et al., 2006; Giedd et al., 1999; Sowell et al., 1999). Accordingly, there is interest in adolescent neural reactivity to the anticipation or delivery of reward (Bjork, Smith, Chen, \& Hommer, 2010; Van Leijenhorst et al., 2010; Eshel, Nelson, Blair, Pine, \& Ernst, 2007; Bjork et al., 2004) as well as in the way rewards are integrated into adolescent behavior through prediction errors (PEs), that is, signals of mismatch between expected and received outcomes (Cohen et al., 2010).

\footnotetext{
${ }^{1}$ University of Reading, ${ }^{2}$ King's College London, ${ }^{3}$ Princeton University
}

adults showed no systematic shifting patterns. The correlation between PE representation and improved performance strengthened with age for activation in ventral and dorsal PFC, ventral striatum, and temporal and parietal cortices. There was a mediallateral distinction in the prefrontal substrates of effective PE utilization between adults and adolescents: Increased utilization of negative PEs, a hallmark of successful performance in the task, was associated with increased activation in ventromedial PFC in adults, but decreased activation in ventrolateral PFC and striatum in adolescents. These results suggest that adults and adolescents engage qualitatively distinct neural and psychological processes during decision-making, the development of which is not exclusively dependent on reward-processing maturation.
These studies point largely toward nonlinear development in ventral striatal reactivity to reward with a peak during adolescence (Galvan, 2010; Ernst \& Fudge, 2009), the impact of which is thought to be exacerbated by the relative immaturity of prefrontal control systems that have been shown to mature into adulthood (Rubia, 2012; Rubia, Hyde, Halari, Giampietro, \& Smith, 2010; Christakou, Halari, et al., 2009; Rubia, Smith, Taylor, \& Brammer, 2007; Rubia et al., 2000, 2006). Characteristically, both ventral PFC activation (Cohen et al., 2010; Galvan et al., 2006) and its functional connectivity with the ventral striatum (VS) have been shown to increase with developmental improvements in reward utilization (Christakou, Brammer, \& Rubia, 2011).

Reward-related decision-making has been studied widely with the Iowa gambling task (IGT; Bechara, Damasio, Damasio, \& Anderson, 1994), a test of decision-making when faced with a mix of ambiguous positive and negative rewards. The task requires forgoing highly rewarding options that nevertheless lead to long-term losses (risky/ disadvantageous choices), in favor of more modest rewards that accumulate into long-term gains (safe/advantageous choices). Adolescent performance in the IGT is independent of working memory or motor inhibition development (Hooper, Luciana, Conklin, \& Yarger, 2004) and improves with age (Van Duijvenvoorde, Jansen, Visser, \& Huizenga, 
2010; Huizenga, Crone, \& Jansen, 2007; Blair, Colledge, \& Mitchell, 2001). Specifically, in appropriate IGT variants, adolescents show increased approach behavior (more safe choices) compared with children or adults; however, avoidance behavior shows linear maturation, as risky choices reduce into adulthood (Cauffman et al., 2010; Crone \& Van der Molen, 2007). Recent evidence describes a decrease in task performance of peripubescent individuals relative to both children and older adolescents, followed by a linear improvement into adulthood (Smith, Xiao, \& Bechara, 2012). The brain processes underlying this developmental performance profile have been, to date, only indirectly inferred.

Less still is known about mechanisms that shape decisionmaking but are only peripherally related to reward processing. Enhanced exploratory drive and reduced risk-related anxiety have been described in both human and animal adolescents (Adriani \& Laviola, 2004; Wills, Vaccaro, \& McNamara, 1994). These characteristics can significantly affect real-world decision-making, by driving exploration irrespective of available rewards or dampening the effects of negative consequences in high-vigilance or ambiguous situations. These considerations may enrich simplistic developmental models restricted to the putative conflict between regulatory and affective networks. This line of thinking requires a dynamic systems approach to the study of development and needs to be informed by the evolutionary imperatives that drive maturational processes.

The immediate testable implication of the rationale outlined above is that adolescent and adult decisionmaking do not necessarily fall along a continuum of behavioral and neural maturation but are characterized by qualitatively distinct neurocognitive mechanisms.

In this study, we examined whether considering an enriched learning model, which accounts not only for the reward-driven aspects of decision-making under ambiguity but also for the increased generalized exploratory characteristics of adolescent behavior, enhances our understanding of both the neural and psychological underpinnings of decision-making maturation. We used a reinforcement learning (RL) model to study the development of decision-making between early adolescence and adulthood in an fMRI-compatible IGT variant. Our model parameterizes aspects of performance relating to sensitivity to previously experienced rewards (decision), rewardindependent choice variability (exploration), and the impact of positive/negative PEs on behavior (outcome evaluation). We used individual differences in these parameters to characterize performance age effects and map the maturation of neural sensitivity to option value during decisions and to PE magnitude during outcome evaluation.

\section{METHODS}

\section{Participants}

Thirty-seven healthy male participants (mean age $=19.6$ years [5.5 years], age range $=11.9-31.2$ years) were recruited through advertisement and word of mouth. The sample included 19 adult participants (mean age $=24.2$ years [3.9 years], age range $=18.2-31.3$ years) and 18 adolescents (mean age $=15.3$ years $[2.1$ years], age range $=$ $11.9-18.0$ years). Ages were normally distributed according to a one-sample Kolmogorov-Smirnov test $(Z=0.798, p=$ $n s)$. Data from the adult sample in this task have been described previously in a different analysis (Christakou, Brammer, Giampietro, \& Rubia, 2009). Given previous evidence for significant sex differences in brain structure, development, and function (including in related tasks and brain regions; Smith, Halari, Giampetro, Brammer, \& Rubia, 2011; Rubia et al., 2010; Christakou, Halari, et al., 2009; Tranel, Damasio, Denburg, \& Bechara, 2005; Giedd et al., 1999), only male participants were included in the study to increase the homogeneity of the results. All participants were right-handed, as assessed using the Edinburgh Handedness Inventory (Oldfield, 1971; mean laterality quotient $=90.69[14.49]$ ). Participant IQ was estimated with the Wechsler Abbreviated Scale of Intelligence (Harcourt Assessment; mean IQ = 116 [11]) and was unaffected by age $(r=-0.174, p=n s)$. Exclusion criteria were psychiatric or neurological disorders, learning disability, current or past drug abuse, head injury, and psychotropic medication. Participants gave written informed consent; adolescent participants were accompanied by at least one parent or guardian who also gave their written informed consent. All participants received $\& 30$ compensation for their participation and were reimbursed for travel expenses. The study was approved by the local research ethics committee.

\section{Gambling Task}

Our fMRI variant of the IGT (Figure 1) has been described in detail previously (Christakou, Brammer, et al., 2009). Briefly, participants were presented with four decks of cards (labeled A, B, C, and D) on a computer screen and were asked to select any one of the decks by pressing with their right hand one of four buttons, arranged horizontally on an MR-compatible button box to correspond with the four decks, the position of which was fixed for each participant for the duration of the session. They were administered 80 presentations of the decks, with the instruction to try to win as much money as possible and lose as little as possible; the only explicit information participants received about the structure of the task was that after a choice sometimes they would win and sometimes they would lose. They were told that their final score would determine how much out of a maximum of $£ 30$ they would receive as compensation (in reality, they all received $\$ 30$ after testing). Participants were unaware of how many trials they would perform or how long the testing session would last. Decks A and B gave relatively large gains (£190, £200, or £210) but even larger losses (£240, 250 , or $£ 260$ ), whereas Decks $C$ and D gave small gains $(£ 90, £ 100$, or $£ 110)$ but even smaller losses ( $\$ 40$, 
Figure 1. Task schematic. On each trial, participants were required to choose one of four decks by pressing the spatially corresponding button on an MR-compatible button box. The choice (RT) was followed by an anticipation period (6 sec) before the outcome of the decision was revealed (feedback), staying on screen for $3 \mathrm{sec}$. The trial ended with a blank screen that took the total trial duration to $15 \mathrm{sec}$. Just as in the original IGT, participants started off with a "loan" of 2000 points on which they could build; this allowed the accommodation of consecutive losses. The loan (red bar) and the current running total (green bar) were present at the bottom of the task display.

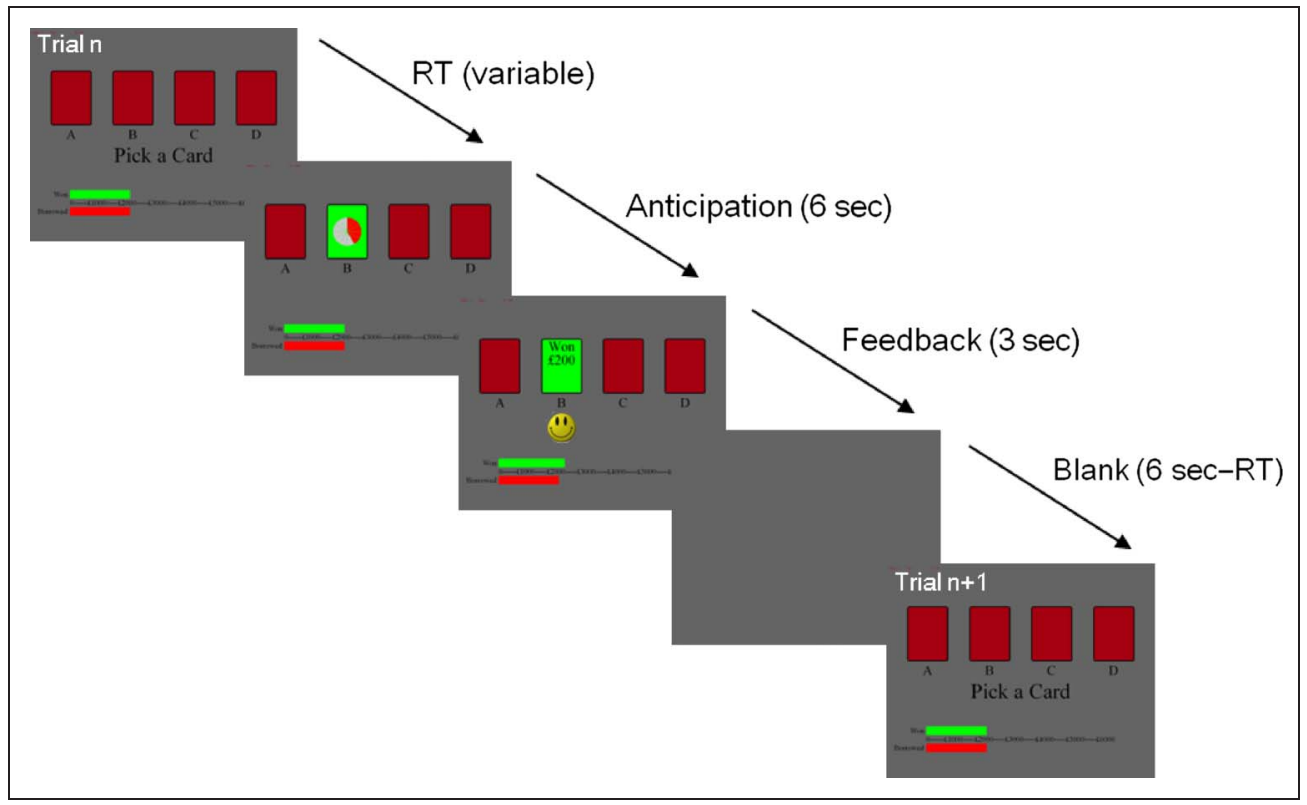

$£ 50$, or $£ 60$ ). There was a 50\% probability of winning or losing on each deck. Consequently, Decks A and B were disadvantageous (also referred to as "risky") because they led to a net loss on average, whereas Decks C and D were advantageous (also referred to as "safe") because they led to a net gain.

Performance on the task is summarized by the subject's advantageous preference ratio, that is, the number of cards picked from the advantageous decks $(C+D)$ divided by the total number of cards picked $(\mathrm{A}+\mathrm{B}+$ $\mathrm{C}+\mathrm{D}$; i.e., ratio $=(\mathrm{C}+\mathrm{D}) /(\mathrm{A}+\mathrm{B}+\mathrm{C}+\mathrm{D}))$. This ratio is proportional to the "net score" $((\mathrm{C}+\mathrm{D})-(\mathrm{A}+\mathrm{B}))$ often used when quantifying performance in the IGT (Bechara et al., 1994), without giving negative values. A preference ratio above 0.5 denotes preference for the advantageous relative to the disadvantageous decks. A ratio below 0.5 denotes perseveration with disadvantageous choices despite accumulating losses. The effect of age on performance was assessed using Pearson's correlation tests (Age $\times$ Preference ratio).

In designing the task trials, we temporally separated the choice response from its outcome, hemodynamically decoupling the moment of decision and the moment of outcome evaluation, allowing us to examine each separately. Following each choice, the deck chosen by the participant was superimposed with a wheel divided into 12 equal segments; every $0.5 \mathrm{sec}$ each consecutive segment filled with color, counting down to outcome presentation. Each trial was consequently divided into (a) the choice phase, from the moment of presentation of the four decks until the execution of the choice (RT to button-press); (b) a 6-sec delay between choosing a deck and being presented with the outcome (win or loss); and (c) the outcome evaluation phase, a 3-sec presentation of the outcome on screen. Trials lasted $15 \mathrm{sec}$, ending with a blank screen after outcome presentation, a period which served as an implicit baseline in the fMRI analysis. The maximum time allowed for a response was $6 \mathrm{sec}$. If no response was registered within that time, the trial progressed directly to the blank screen for 9 sec (making up the total trial time of $15 \mathrm{sec}$ ). Omitted trials were excluded from the analysis. The length of each intertrial interval was determined by the RT, which jittered trial events so as to maintain a 15 -sec trial duration. Because these manipulations significantly lengthened the duration of trials and of the whole task session compared with other behavioral variants, we used 80 trials instead of the typical 100 (Lawrence, Jollant, O’Daly, Zelaya, \& Phillips, 2009; Bechara et al., 1994).

Before testing, participants were acclimatized to the scanner environment in a "mock" scanner, in which they practiced the task they were going to perform in an environment similar to the scanner facility. This practice session consisted of 12 trials that presented equal payoffs across all decks, and this difference between the training and experimental sessions was made explicit to participants. After completion of the experimental session, participants were asked whether they had picked more cards from any particular deck(s) or whether they had avoided any particular deck(s) and why. Nine of 19 adults (47.37\%) and 2 of 18 adolescents (11.11\%) were considered aware of the general contingencies of the task, as they could report that Decks A and/or B would "mostly lose" or "made you lose more money than you won" and/or that Decks C and/or D would "mostly win" or "let you win more money than you lost."

\section{Temporal Difference RL Model}

Given trial-and-error learning in the task, previous evidence of differential impact of positive and negative outcomes on 
performance (Christakou, Brammer, et al., 2009) and the hypothesized impact of adolescent exploratory behavior on decision-making, we constructed a temporal difference (TD) RL model (Sutton \& Barto, 1998) that included separate learning rates for positive and negative PEs (Niv, Edlund, Dayan, \& O'Doherty, 2012; Frank, Moustafa, Haughey, Curran, \& Hutchison, 2007) as well as a choice kernel ("stickiness" parameter) that captured individual choice variability independently of reward history.

We denote the chosen deck on trial $t$ by $c_{t}$ and the reward feedback by $r_{t}$. We posit the following "softmax" probability distribution over choices (Sutton \& Barto, 1998):

$$
P\left(c_{t}=c\right)=\frac{e^{m_{t}(c)}}{\sum_{j} e^{m_{t}(j)}}
$$

where we refer to $m_{t}(c)$ as the preference for $c$ and $j$ enumerates over all four possible choices. The preference is decomposed into two contributing factors, the value $v_{t}(c)$ (reflecting the reward history) and the stickiness $u_{t}(c)$ (reflecting the immediate choice history independently of reward; Gershman, Pesaran, \& Daw, 2009; Lau \& Glimcher, 2005):

$$
m_{t}(c)=\beta v_{t}(c)+\omega u_{t}(c)
$$

where $\beta$ and $\omega$ are inverse temperature parameters that control the influence of each factor on the preference function. We refer to $\beta$ as outcome sensitivity and $\omega$ as choice sensitivity.

The value function is updated according to the following TD rule:

$$
v_{t+1}\left(c_{t}\right)= \begin{cases}v_{t}\left(c_{t}\right)+\alpha^{+} \delta_{t} & \text { if } \delta_{t}>0 \\ v_{t}\left(c_{t}\right)+\alpha^{-} \delta_{t} & \text { if } \delta_{t}<0\end{cases}
$$

where $\delta_{t}=r_{t}-v_{t}\left(c_{t}\right)$ is the PE and $\alpha^{+}$and $\alpha^{-}$are learning rate parameters for positive and negative PEs, respectively.

The stickiness captures residual autocorrelation between choices that cannot be explained by reward history and is defined as follows:

$$
u_{t+1}(c)=\left\{\begin{array}{l}
1 \text { if } c_{t}=c \\
0 \text { otherwise }
\end{array}\right.
$$

Thus, the model has four parameters: $z=\left\{\beta, \omega, \alpha^{+}, \alpha^{-}\right\}$.

In psychological terms, $\beta$ parameterizes the influence of past decision outcomes on future behavior (which we refer to as outcome sensitivity), and $\omega$ parameterizes the extent to which each participant tended to stay with or shift responding between decks regardless of the outcomes of decisions (we refer to this as choice sensitivity). The learning rates parameterize the extent to which learning from better than expected $\left(\alpha^{+}\right)$or worse than expected $\left(\alpha^{-}\right)$outcomes drives future individual choice behavior.

\section{Model Fitting}

We found the maximum a posteriori parameter estimates for each participant separately using gradient ascent on the posterior score:

$$
P(z \mid D) \propto P(D \mid z) P(z)
$$

where $D=\{c, r\}$ is the observed choices and rewards. The likelihood $P(D \mid z)$ is the softmax probability of the observed choices (Equation 1). To avoid degenerate parameter estimates due to the small number of trials and the noisiness of the data, we used the following as the prior $P(z)$ :

$$
\begin{gathered}
\beta \sim \operatorname{Gamma}(2,1) \\
\omega \sim N(0,1) \\
\alpha^{+} \sim \operatorname{Beta}(1.2,1.2) \\
\alpha^{-} \sim \operatorname{Beta}(1.2,1.2)
\end{gathered}
$$

In fitting the model with stickiness, we aimed for the choice stickiness parameter to capture residual variance not explained by reward history. To this end, we fit the model in two stages, first fitting the reward sensitivity and learning rate parameters (with choice stickiness set to 0 ) and then holding these fixed while fitting the choice stickiness.

To test whether the enhanced RL model (Model 1) was justified by the data, we compared its fit with that of two simpler models: a model that included a single learning rate and stickiness (Model 2) and a simple TD model that included a single learning rate and no stickiness (Model 3). Furthermore, we also examined the relative fit of our model in the adult and adolescent subgroups separately to ensure that comparisons of model parameters between groups and across the age range were justified.

Model fit was assessed by calculating, for each model, the log Bayes factor (Kass \& Raftery, 1995) relative to a chance (null) model in which each deck is chosen with equal probability. The log Bayes factor balances data fit against model complexity. Under a uniform prior over models, the log Bayes factor is directly proportional to the log posterior probability of a model. Log Bayes factors greater than 2.3 (a 10:1 ratio) are conventionally considered strong evidence for a model (Jeffreys, 1961). Because the log Bayes factor involves an intractable integral over parameter values, we used the Laplace approximation (Kass \& Raftery, 1995), which assumes that the posterior over parameters is Gaussian around the mode (using the Bayesian information criterion approximation instead did not qualitatively change the results).

To allow for individual variability in model fit, we treated the log Bayes factors across participants as random effects (for examples of this approach, see Simon \& Daw, 2011; 
Stephan, Penny, Daunizeau, Moran, \& Friston, 2009). Differences across groups and models were assessed using nonparametric statistics (Wilcoxon rank sum and signed rank tests, respectively; Table 1).

Model comparison showed that participants' choice behavior was best explained by Model 1, the full model that included both choice stickiness and two separate learning rates for learning from positive and negative PEs. Table 1 details the model comparison statistics for all participants and for each group on its own. Moreover, when examining the models' fit on the level of each individual participant, Model 1 resulted in the best fit for 30 of 37 participants (15 of 19 adults and 15 of 18 adolescents). We therefore used Model 1 for all subsequent analyses of behavioral and neural data.

\section{Use of Model Parameters to Characterize Individual Performance}

Correlations between performance or brain activation and model parameter estimates as well as the effect of age on parameter estimates were assessed with Pearson's correlation tests. For categorical comparisons between adults and adolescents (i.e., age $<18$ years), we used

Table 1. Model Comparisons

\begin{tabular}{|c|c|c|c|}
\hline & All Participants & Adults & Adolescents \\
\hline \multicolumn{4}{|c|}{ A. Model Log Bayes Factors versus Chance } \\
\hline \multicolumn{4}{|l|}{ Model 1} \\
\hline Median & 4.71 & 6.81 & 5.64 \\
\hline 25th-75th percentile & $0.81-9.83$ & $0.40-12.52$ & $0.39-10.04$ \\
\hline$p$ & $<.001$ & $<.001$ & $<.001$ \\
\hline Signed rank & 63 & 12 & 9 \\
\hline$Z$ & -5.92 & -3.34 & -3.33 \\
\hline \multicolumn{4}{|l|}{ B. Model Comparisons } \\
\hline \multicolumn{4}{|l|}{ Model 1 versus Model 2} \\
\hline Median & 4.71 & 6.81 & 5.64 \\
\hline 25 th-75th percentile & $0.81-9.83$ & $0.40-12.52$ & $0.39-10.04$ \\
\hline$p$ & $<.001$ & $<.001$ & $<.001$ \\
\hline Signed rank & 63 & 12 & 9 \\
\hline$Z$ & -5.92 & -3.34 & -3.33 \\
\hline \multicolumn{4}{|l|}{ Model 1 versus Model 3} \\
\hline Median & 5.66 & 7.56 & 4.19 \\
\hline 25 th-75th percentile & $1.73-12.10$ & $2.75-18.21$ & $1.08-10.21$ \\
\hline$P$ & $<.001$ & $<.001$ & $<.001$ \\
\hline Signed rank & 52 & 10 & 7 \\
\hline \multirow[t]{2}{*}{$Z$} & -6.02 & -3.42 & -3.42 \\
\hline & Model 1 & Model 2 & Model 3 \\
\hline \multicolumn{4}{|c|}{ C. Adults versus Adolescents } \\
\hline$p$ & $.19(n s)$ & $.28(n s)$ & $.10(n s)$ \\
\hline Signed rank & 298 & 378 & 288 \\
\hline$Z$ & -1.32 & 1.08 & -1.63 \\
\hline
\end{tabular}

(A) Assessment of model fit for the winning model (Model 1) for the whole sample and across the two age subgroups (adults and adolescents). (B) Comparison of the three models considered. (C) Model fit comparisons between adults and adolescents for all three models considered, ensuring that comparisons of model parameter estimates between groups and across the age-range are justified. 
ANOVA; deviations from zero were assessed with onesample $t$ tests.

\section{fMRI}

\section{Acquisition}

Gradient echo echo-planar MRI data were acquired on a GE Signa 3-T system (General Electric, Milwaukee, WI) at the Centre for Neuroimaging Sciences, King's College London, using a semiautomated image quality control procedure. A quadrature birdcage head coil was used for radio-frequency transmission and reception. We acquired 800 T2*-weighted MRIs depicting BOLD contrast in each of 22 noncontiguous planes, covering the whole brain (echo time $=30 \mathrm{msec}$, repetition time $=1.5 \mathrm{sec}$, flip angle $=60^{\circ}$, in-plane resolution $=3.75 \mathrm{~mm}$, slice thickness $=5.0 \mathrm{~mm}$, slice skip $=0.5 \mathrm{~mm}$ ). We also acquired a 43-slice, whole-brain, high-resolution structural scan (gradient-echo EPI; echo time $=40 \mathrm{msec}$, repetition time $=3 \mathrm{sec}$, flip angle $=90^{\circ}$, slice thickness $=3.0 \mathrm{~mm}$, slice skip $=0.3 \mathrm{~mm}$ ), on which we superimposed the activation maps. Quality control was carried out using an automated analysis tool to ensure high quality images (Simmons, Moore, \& Williams, 1999).

\section{Data Analysis}

fMRI data were analyzed with the XBAM software (XBAM v4.1) developed at the Institute of Psychiatry (www.brainmap. co.uk). The software uses a nonparametric permutationbased strategy (rather than normal theory-based inference) to minimize assumptions and uses median rather than mean-based statistics to control for outlier effects. Its most commonly used test statistic is computed by standardizing for individual differences in residual noise before secondlevel multisubject testing using robust permutation-based methods. This allows a mixed-effects approach to analysis, an approach that has been recommended following detailed analysis of the validity and impact of normal theorybased inference in fMRI in a large number of subjects (Thirion et al., 2007). Individual and group-level analyses have been described in further detail elsewhere (Christakou, Brammer, et al., 2009).

The fMRI data were realigned to minimize motionrelated artifacts and smoothed using a Gaussian filter (FWHM 8.82 mm; Bullmore et al., 1999). Time-series analysis of individual participant activation was performed with a wavelet-based resampling method previously described (Bullmore et al., 2001). We first convolved the task epoch of each event of interest (decision and outcome), with two Poisson model functions (delays of 4 and $8 \mathrm{sec}$ ). We used trial-by-trial V values (for the decision) and PE values (for the outcome), interpolating values across trial time points, to convolve each resulting function with the model of the hemodynamic response. Individual activation maps were recalculated by testing the goodness of fit of this convolution with the BOLD time series; the goodness-of-fit calculation used the ratio of the sum of squares of deviations from the mean intensity value due to the model (fitted time series) divided by the sum of squares due to the residuals (original time series minus model time series). This statistic, the sum of squares (SSQ) ratio, was used in further analyses. Individual maps then represent brain areas where BOLD activation during decisions or outcomes scaled with the magnitude of $\mathrm{V}$ or PE, respectively (for convenience, we shorthand this statistic as the fidelity of the V or PE neural representation). Using rigid body and affine transformation, the individual maps were registered into Talairach standard space.

Whole-brain regression with age. Whole-brain regression analysis using cluster-level permutation statistics was carried out, identifying brain regions where the fidelity of the representation of $\mathrm{V}$ (during decision) or PE (during outcomes) in the BOLD time series correlated with age. The Pearson's product-moment correlation coefficient was first computed at each voxel in standard space between age and SSQ over all participants. The correlation coefficients were recalculated after randomly permuting the participants' age (but not the fMRI data). Repeating the second step many times (1000 times per voxel, then combining over all voxels) gives the distribution of correlation coefficients under the null hypothesis that there is no association between specific age and specific SSQs. This null distribution was then used to assess the probability of a correlation between age and the fidelity of V or PE representation under the null hypothesis. The critical value of the correlation coefficient at any desired Type I error level in the original (nonpermuted) data can be determined by reference to this distribution. Statistical analysis was extended to cluster level as described by Bullmore et al. (1999). The cluster level probability under the null hypothesis was chosen to set the level of expected Type I error clusters to $<1$ per whole brain.

Regression of age-correlated data masks with performance. We were interested in brain maturation processes that underlie individual differences in performance as indexed by the preference ratio. Therefore, to identify brain areas where age-dependent increases in the fidelity of the representation of $\mathrm{V}$ or $\mathrm{PE}$ were associated with increased performance, we first extracted 3-D masks of age-correlated SSQs (see Whole-brain Regression with Age section) and then used these masks to confine a voxel-wise regression analysis with performance (preference ratio) using the same method. This analysis yielded areas where individual differences in age and performance interacted in their impact on brain representation of value during decisions or PE during outcomes. Given differences in the relationship between age and performance in adults and adolescents (Results section, Maturation of Task Performance) we also performed this analysis for each age group separately. 
To further characterize age effects on the relationship between neural PE representation and performance, we examined age group differences in the coefficient of correlation between PE-modulated brain activation and preference ratio. For each group independently, the average Pearson's product-moment correlation coefficient between preference ratio and SSQs was computed, and the difference in correlation between the two groups was calculated. To determine the significance of this difference, the appropriate null distribution was generated by randomly permuting participants and their preference ratio between the groups (without replacement) and scrambling any group differences. For each of the 1000 permutations, the difference in correlation between the scrambled groups was calculated, and the resulting values were combined over all voxels to produce a maskwide null distribution of differences in correlation. Testing was then extended to cluster level, with the cluster probability under the null hypothesis chosen to set the level of expected Type I error clusters at less than one.

Correlations of brain activation with model parameters. To test for correlations between V- or PE-modulated BOLD activation and model parameter estimates, we extracted the cluster-wise goodness of fit statistic (SSQ ratios) for each participant. This statistic represents the fidelity of neural V or PE representation in the given cluster (and not magnitude of activation per se). We then performed Pearson's correlations with model parameters correcting for multiple comparisons.

\section{RESULTS}

\section{Behavioral Results}

\section{Maturation of Task Performance}

There was a significant improvement with age in task performance (proportion of safe or advantageous choices made across the whole testing session, the preference ratio), $r(35)=0.448, p<.005$, consistent with previous studies showing developmental improvements in performance of the IGT (Smith, Xiao, et al., 2012; Cauffman et al., 2010). On the basis of previous evidence that maturational improvements in performance peak by early adulthood (Cauffman et al., 2010), we examined the possibility that adolescents (age $<18$ years) contributed to the observed age-related improvement of performance more than adults. Indeed, this improvement was significant in adolescents, $r(16)=0.541, p<.05$, whereas in adults there was no longer a relationship between age and performance, $r(17)=0.083, p=n s$ (Figure 2).

As can be seen in Figure 2, adult participants showed significantly increased preference for the advantageous options across the four 20-trial blocks of the task, whereas adolescent participants showed no changes in preference across the testing session (Block $\times$ Group repeatedmeasures ANOVA: significant main effect of Group, $F(1$,

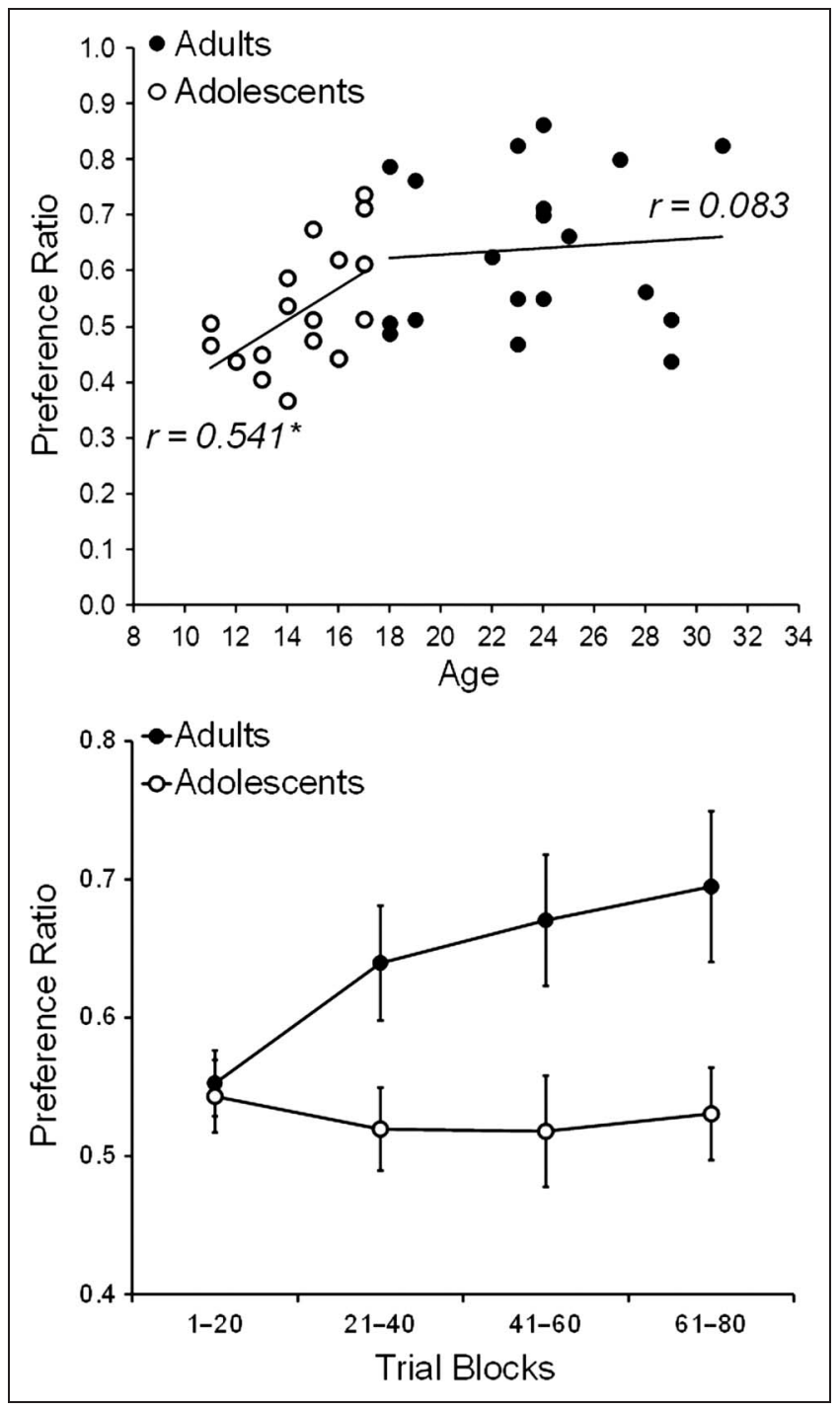

Figure 2. The main performance measure, preference ratio, refers to the ratio of advantageous (or safe) choices over all choices made across the testing session, here plotted against participant age. Performance improved with age during adolescence and stabilized in adulthood (two-tailed Pearson's $r$ coefficient reported for the adolescent and adult subgroups; *significant at the $p<.05$ level).

$35)=7.285, p<.05$; no main effect of Block, $F(3$, $105)=1.779, p=n s$; significant Block $\times$ Group interaction, $F(3,105)=2.865, p<.05)$.

We finally note that there was no difference in (the very few) omission errors between the groups, $t(35)=$ $-1.997, p=n s$. Omitted trials were excluded from the analyses.

\section{Influence of Model Parameters on Performance and Effects of Age}

Both outcome sensitivity and choice sensitivity increased with age across the whole sample, $r(35)=0.360, p<.05$ and $r(35)=0.455, p<.01$, respectively, and the two age subgroups (adults and adolescents) differed significantly 
in both parameters, $F(1,35)=4.303, p<.05$ and $F(1$, $35)=4.855, p<.05$, respectively. Increases in both parameters across the sample were associated with improved performance, $r(35)=0.704, p<.001$ and $r(35)=0.359$, $p<.05$, respectively (Figure $3 \mathrm{~A}$ and $\mathrm{C}$ ). The effect of outcome sensitivity on performance survived correction for age (tested with a correlation entering age as a covariate: $r(34)=0.650, p<.001)$, indicating that developmental influences on sensitivity to the nature of reward outcomes account for only some of the variance in performance in this population (notably there were no differences between the adolescent and adult subgroups in the relationship of outcome sensitivity and age; comparison of Fisher $z$-transformed correlation coefficients: $z=-0.26, p=n s$ ). However, the effect of choice sensitivity on performance did not survive correction for age, $r(35)=0.195, p=n s$, emphasizing the developmental nature of its relationship with increased advantageous choices. Specifically, adolescent participants as a group showed negative choice sensitivity, indicating a tendency to shift from previous options regardless of outcome, $t(17)=-3.735, p<.005$. In contrast, adult participants showed no deviation from choice sensitivity of zero, $t(18)=-0.120, p=n s$, indicating that their choices were governed primarily by the outcomes of previous choices (Figure 3; see Methods section for description of the model parameters).

Individual sensitivity to positive and negative PEs (quantified by the learning rate, an indication of the strength of the impact of current rewards on future behavior) had opposite effects on performance: high sensitivity to positive PEs (that is to better than expected outcomes) was associated with low ratios of advantageous choices, $r(35)=-0.446, p<.01$, whereas high sensitivity to negative PEs (to worse than expected outcomes) was associated with high ratios of advantageous choices, $r(35)=0.800$, $p<.001$. This result indicates that learning from worse than expected outcomes is beneficial whereas learning from better than expected outcomes is detrimental in this task. Both associations were weakened but survived after correction for age, $r(34)=-0.354, p<.05$ and $r(34)=$ $0.789, p<.001$, respectively, indicating a partial developmental contribution. The maturation profile of both parameters echoed their relationship with performance: sensitivity to positive PEs decreased with increasing age across the whole sample, $r(35)=-0.328, p<.05$. Conversely, sensitivity to negative PEs increased with age, but only across adolescence, $r(16)=0.567, p<.05$, not in adulthood, $r(17)=0.093, p=n s$, echoing the pattern of developmental improvement in performance.

The summary measure of the difference in learning rates $\left(\Delta_{\alpha}=\alpha^{+}-\alpha^{-}\right)$is shown in Figure 3E across the whole sample. More negative $\Delta_{\alpha}$ was predictive of better choice performance in the task, $r(35)=-0.844, p<$ .001 . More negative $\Delta_{\alpha}$ was characteristic of adult participants, $t(18)=-4.17, p<.001$, whereas adolescents did not show differential sensitivity to positive and negative PEs, $t(17)=-0.99, p=n s$ (Figure $3 \mathrm{~F}$ ).
In a parallel analysis to further probe qualitative differences in the performance of adults and adolescents, we split each age group into performance subgroups on the median of their preference ratio. Low-performing adults $(n=9)$ showed significantly decreased outcome sensitivity compared with high-performing adults $(n=10)$, $t(17)=4.46, p<.001$, and did not exhibit increased sensitivity to negative compared with positive PEs as indicated by a $\Delta \alpha$ that was not significantly lower than zero, $t(9)=-0.61, p=n s$. However, even low-performing adults demonstrated neutral choice sensitivity ( $\omega$ was not significantly different to zero: $t(8)=-1.17, p=n s$ ). Conversely, high-performing adolescents $(n=9)$ showed increased sensitivity to negative PEs $(\Delta \alpha$ was significantly lower than zero: $t(8)=-5.46, p<.01)$, whereas lowperforming adolescents $(n=9)$ showed increased sensitivity to positive PEs ( $\Delta \alpha$ was significantly lower than zero: $t(8)=4.05, p<.005)$. High-performing adolescents did not benefit from increased outcome sensitivity compared with low-performing adolescents, $t(17)=-0.17, p=n s$, nor, importantly, did they show reduced exploratory tendencies (choice sensitivity was significantly lower than zero in both high- and low-performing adolescent subgroups: $t(8)=-2.60, p<.05$ and $t(8)=-2.55, p<$ .05 , respectively).

\section{Neuroimaging Results}

We used trial-by-trial regressors of decision values and PEs with a whole-brain voxel-wise correlation with age (as described in the Methods section). This analysis identified brain areas where the utilization of decision values (during the decision phase) and PEs (during the outcome evaluation phase) matures linearly with age across the sample. Although significantly more complex dynamics determine structural and functional brain maturation, we note that, in this sample and experimental setup, there were no differences in the activation maps thus derived when examining non-linear (quadratic) effects of age.

We further interrogated these age-correlated areas ("age mask") to identify regions associated with improvements in task performance. Given the difference in the relationship between performance and age in the adult and adolescent subgroups, we repeated the voxel-wise correlation of the age mask with preference ratio separately for the two age groups and compared the voxel wise correlation coefficient with preference ratio.

\section{Decision Phase: Representation of Value}

During the decision phase, activation in dorsal PFC, ventromedial PFC (vmPFC), and ventrolateral PFC (vlPFC), temporal and postcentral parietal cortex, and cerebellum showed increased sensitivity with maturing age to the value of upcoming decisions (Table $2 \mathrm{~A}$ ). A voxel-wise correlation of the age mask with preference ratio showed that value representation in dorsolateral premotor cortex 

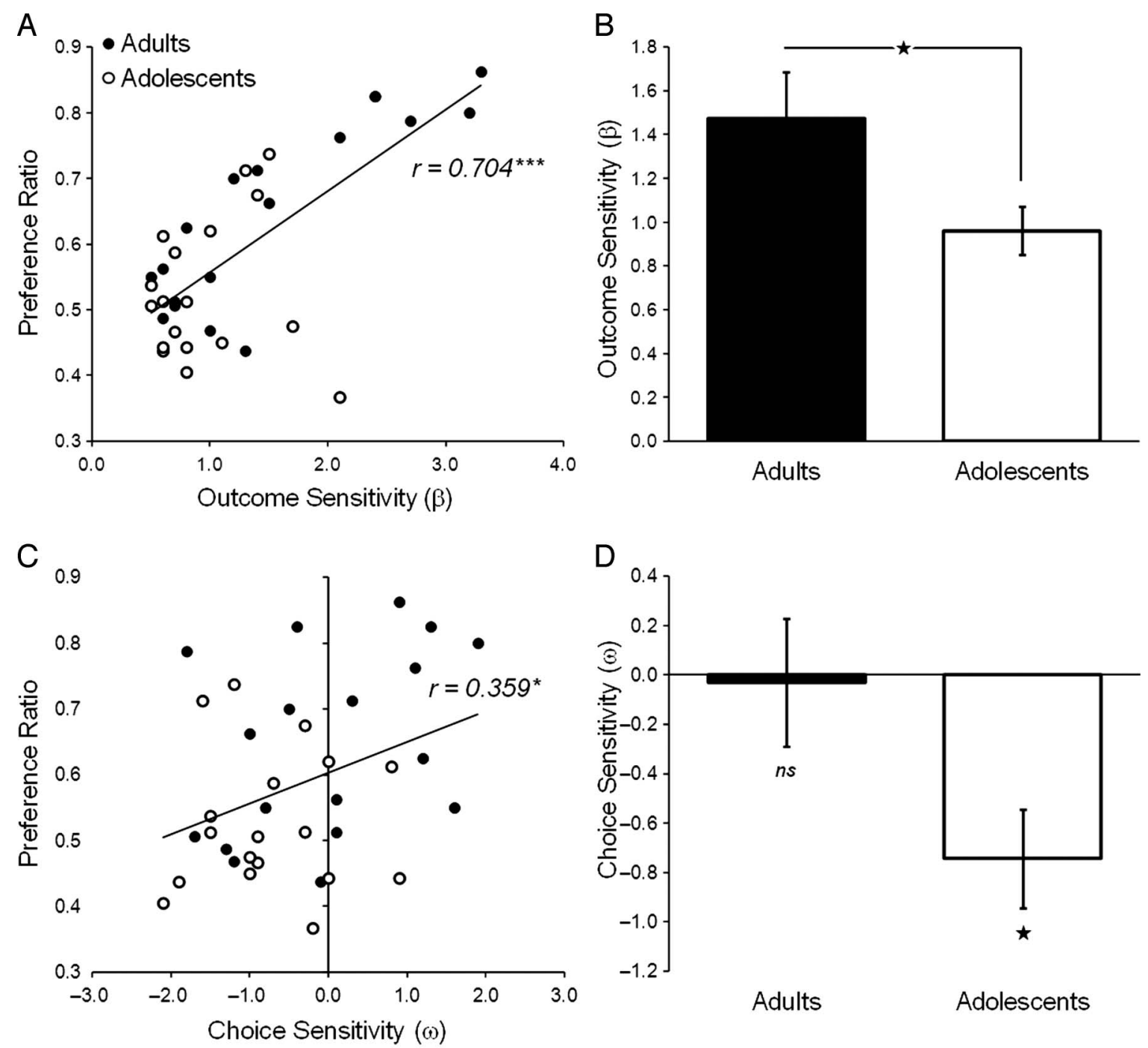

Adults

Adolescents

E

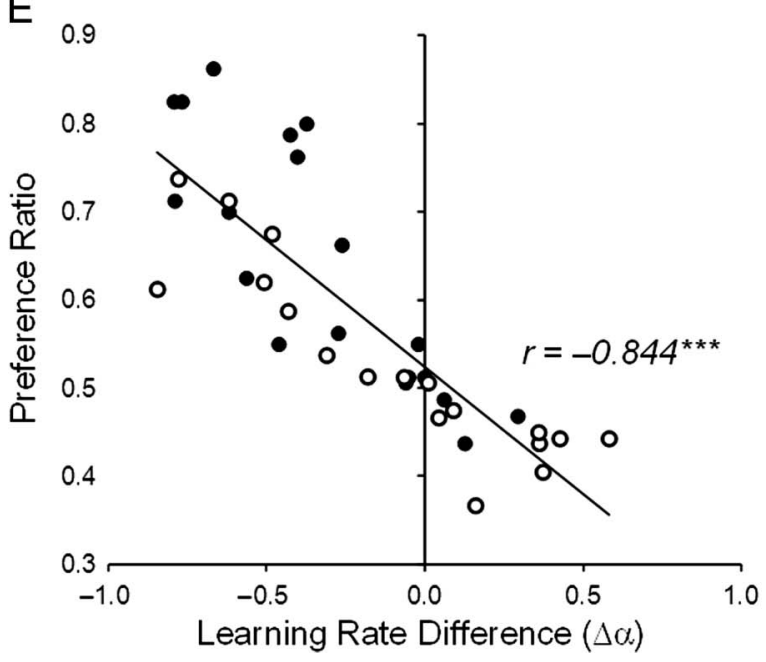

F

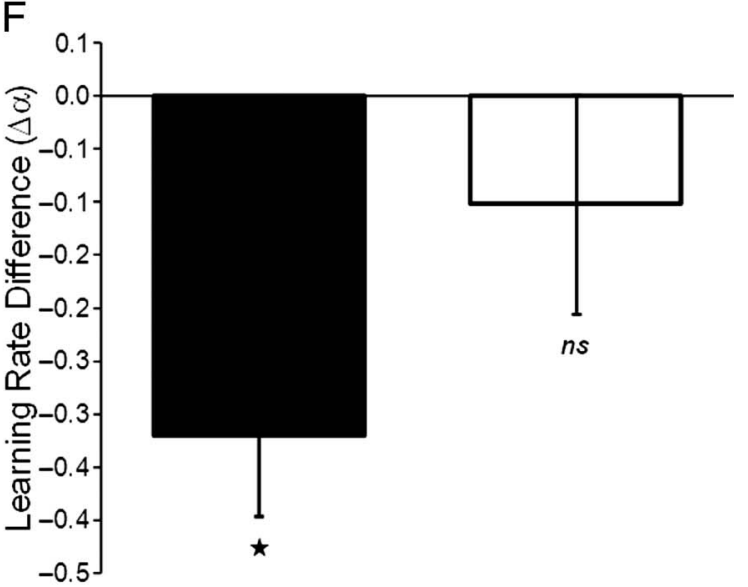

Adults

Adolescents

Figure 3. The relationship between parameter estimates and performance (correlation with preference ratio, left) and group mean parameter estimates for adults and adolescents (right). Two-tailed Pearson's $r$ coefficient reported for the correlation between model parameter and preference ratio (A, C, and E). Between group ANOVA (B) and $t$ tests of deviations from zero (D and F) reported as follows: $n s$, not significant; *, significant at the $p<.05$ level; ***, significant at the $p<.001$ level. 
Table 2. Neural Representation of Value during Decisions

\begin{tabular}{|c|c|c|c|c|c|c|}
\hline \multirow[b]{2}{*}{ Cluster Location (BA) } & \multirow[b]{2}{*}{ Side } & \multicolumn{3}{|c|}{ Talairach Coordinates } & \multirow[b]{2}{*}{ Probability } & \multirow[b]{2}{*}{ Size } \\
\hline & & $x$ & $y$ & $z$ & & \\
\hline \multicolumn{7}{|l|}{$A$. } \\
\hline Superior frontal gyrus (BA 6) & $\mathrm{L}$ & -14 & 33 & 53 & .000 & 25 \\
\hline Middle frontal gyrus (BA 6) & $\mathrm{L}$ & -29 & 11 & 64 & .000 & 58 \\
\hline Superior frontal gyrus (BA 9) & $\mathrm{R}$ & 14 & 52 & 37 & .000 & 13 \\
\hline Medial frontal gyrus (BA 11) & $\mathrm{R}$ & $11 / 2$ & $63 / 55$ & $-40 /-18$ & .000 & 107 \\
\hline Inferior frontal gyrus (BA 44) & $\mathrm{R}$ & 58 & 11 & 15 & .001 & 11 \\
\hline $\begin{array}{l}\text { Inferior frontal gyrus (BA 47); } \\
\text { superior temporal gyrus (BA 38) }\end{array}$ & $\mathrm{L}$ & $-43 /-43$ & $33 / 25$ & $-29 /-17$ & .000 & 108 \\
\hline Inferior temporal gyrus (BA 21) & $\mathrm{L}$ & -65 & -4 & -18 & .001 & 11 \\
\hline Superior temporal gyrus (BA 39) & $\mathrm{L}$ & -43 & -52 & 20 & .000 & 16 \\
\hline Parahippocampal gyrus (BA 35) & $\mathrm{L}$ & -29 & -22 & -18 & .000 & 19 \\
\hline Postcentral gyrus (BA 1) & $\mathrm{L}$ & -54 & -22 & 53 & .000 & 27 \\
\hline Cuneus (BA 18) & $\mathrm{L}$ & -7 & -100 & 9 & .004 & 11 \\
\hline $\begin{array}{l}\text { Cerebellum, posterior lobe, } \\
\text { inferior semilunar lobule }\end{array}$ & $\mathrm{L}$ & -25 & -63 & -40 & .000 & 43 \\
\hline Cerebellum, posterior lobe, declive & $\mathrm{R}$ & 36 & -74 & -18 & .000 & 18 \\
\hline \multicolumn{7}{|l|}{$B}$. \\
\hline Superior frontal gyrus (BA 8) & $\mathrm{L}$ & $-11 /-23$ & $41 / 31$ & $59 / 51$ & .009 & 21 \\
\hline Superior frontal gyrus (BA 6) & $\mathrm{L}$ & $-4 /-10$ & $11 / 8$ & $64 / 65$ & .001 & 48 \\
\hline
\end{tabular}

(A) Clusters where the strength of the representation of $\mathrm{V}$ during decisions was correlated with age across the whole sample. (B) Age-correlated clusters that were also correlated with performance (preference ratio). Only clusters comprising more than 10 voxels are presented. Talairach coordinates shown for the peak of each 3-D cluster; where two coordinates are shown they refer to the peak and the geometric center of the cluster, respectively. Probability: cluster-wise probability correlation of correlation with (A) age or (B) preference ratio. Size: number of activated voxels per 3-D cluster.

(BA 6) was predictive of improvements in performance (Table 2B), but this effect was only contributed by the adult participants and was not significant in the adolescent subgroup.

\section{Outcome Phase: Representation of PE}

During the outcome evaluation phase, two large contiguous clusters showed age-related changes in their sensitivity to the magnitude of PEs during outcome evaluation (Table 3A). They included vmPFC, dorsolateral PFC (dlPFC), VS, precentral cortex, inferior temporal cortex, superior parietal cortex, and cerebellum.

Across the whole sample, age-related changes in PE representation in overlapping dorsolateral, precentral, parietal, occipital, and cerebellar clusters were associated with improvements in performance. In addition, adults showed a positive correlation between performance and activation in the vmPFC, whereas adolescents showed a negative correlation between performance and activation in vlPFC, VS, putamen and subgenual ACC (Table 3B).
Statistically significant differences between the groups in the correlation coefficient between PE-modulated activation and performance were found in vmPFC, subgenual ACC, VS, and visual cortex (Figure 4).

\section{Association of Model Parameters with Frontostriatal Activation}

We showed above that the difference in sensitivity to positive and negative PEs $(\Delta \alpha)$ matures with age and predicts performance. In addition, we showed that vmPFC and dlPFC activation, previously implicated in performance of the task (Christakou, Brammer, et al., 2009), matures to reflect the magnitude of PEs during outcome evaluation in parallel to developmental improvements in performance (Table 3B, Whole Sample). Consequently, we sought to test the predicted association between $\Delta \alpha$ and $\mathrm{PE}$ representation in the vmPFC and dlPFC during outcome evaluation. As predicted by our previous work, there was a weak but significant negative correlation between PE representation in the $\mathrm{VmPFC}$ and $\Delta \alpha, r(35)=-0.315$, 
$p<.05$ one-tailed (Figure 5), with an even stronger correlation in dlPFC, $r(35)=-0.534, p<.001$. These correlations indicate that the higher the fidelity of the PE representation was in the $\mathrm{vmPFC}$, the more behavior was affected by negative PEs as compared with positive ones. Although the causal direction of this association is not testable in our data, it suggests a close relationship be- tween neural and behavioral maturation in the utilization of PEs. We further note that this relationship was only significant in the vmPFC for adult participants, $r(17)=$ $-0.449, p<.05$ one-tailed (Figure 5), and not for adolescents, $r(16)=0.182, p=n s$ one-tailed (Figure 5), but was significant for both groups in the dlPFC (adults: $r(17)=$ $-0.548, p<.05$; adolescents: $r(16)=-0.528, p<.05)$.

Table 3. Neural Representation of PEs during Outcome Evaluation

\begin{tabular}{|c|c|c|c|c|c|c|}
\hline \multirow[b]{2}{*}{ Cluster Location (BA) } & \multirow[b]{2}{*}{ Side } & \multicolumn{3}{|c|}{ Talairach Coordinates } & \multirow[b]{2}{*}{ Probability } & \multirow[b]{2}{*}{ Size } \\
\hline & & $x$ & $y$ & $z$ & & \\
\hline \multicolumn{7}{|l|}{ A. } \\
\hline $\begin{array}{l}\text { Cerebellum (pyramis, declive, culmen); } \\
\text { lingual gyrus (BA 18, 17); middle occipital } \\
\text { gyrus (BA 18, 19); posterior cingulate } \\
\text { (BA 30); cuneus (BA 18, 7); precuneus } \\
\text { (BA 31, 19, 7) }\end{array}$ & $\mathrm{R}$ & 18 & -74 & 31 & .002 & 972 \\
\hline $\begin{array}{l}\text { Cerebellum (inferior semilunar lobule, uvula, } \\
\text { tuber); medial frontal gyrus (vmPFC/BA 11); } \\
\text { precentral gyrus (BA 6); insula (BA 40); inferior } \\
\text { frontal gyrus (BA 44, 9); postcentral gyrus } \\
\text { (BA 40, 3); inferior parietal lobule (BA 40) }\end{array}$ & $\mathrm{L}$ & -54 & -22 & 20 & .001 & 946 \\
\hline \multicolumn{7}{|l|}{$B$. } \\
\hline \multicolumn{7}{|l|}{ Whole sample } \\
\hline $\operatorname{vmPFC}(\mathrm{BA} \mathrm{11})$ & $\mathrm{L}$ & -4 & 44 & -24 & .068 & 25 \\
\hline dlPFC (BA 9); precentral gyrus (BA 6) & $\mathrm{L}$ & -40 & 7 & 31 & .017 & 55 \\
\hline Cerebellum; occipital cortex & $\mathrm{R}$ & 4 & -74 & -13 & .000 & 776 \\
\hline Postcentral gyrus (BA 40) & $\mathrm{L}$ & -40 & -30 & 53 & .000 & 279 \\
\hline \multicolumn{7}{|l|}{ Adults } \\
\hline vmPFC (BA 11) & $\mathrm{L}$ & -4 & 41 & -24 & .000 & 11 \\
\hline dlPFC (BA 9); precentral gyrus (BA 6) & $\mathrm{L}$ & -51 & 0 & 26 & .000 & 17 \\
\hline Postcentral gyrus, parietal cortex (BA 3) & $\mathrm{L}$ & -40 & -26 & 59 & .000 & 143 \\
\hline Cerebellum & $\mathrm{L}$ & -18 & -67 & -29 & .000 & 400 \\
\hline Cerebellum & $\mathrm{R}$ & 25 & -85 & 9 & .000 & 35 \\
\hline Cerebellum & $\mathrm{R}$ & 11 & -70 & 26 & .000 & 32 \\
\hline \multicolumn{7}{|l|}{ Adolescents } \\
\hline vlPFC (BA 47) & $\mathrm{L}$ & -25 & 33 & -24 & .000 & 20 \\
\hline VS & $\mathrm{L}$ & -14 & 11 & -13 & .000 & 14 \\
\hline Subgenual ACC/OFC (BA 25) & $\mathrm{R}$ & 11 & 26 & -18 & .000 & 34 \\
\hline dlPFC (BA 9) & $\mathrm{L}$ & -40 & 7 & 31 & .000 & 31 \\
\hline Precentral gyrus (BA 6) & $\mathrm{L}$ & -25 & -19 & 59 & .000 & 107 \\
\hline Primary visual (peristriate) cortex (V2, V3) & $\mathrm{L}$ & -7 & -85 & -7 & .000 & 586 \\
\hline
\end{tabular}

(A) Clusters where the strength of the representation of PE during outcome evaluation was correlated with age across the whole sample. (B) Agecorrelated clusters that were also correlated with performance (preference ratio) for the whole sample and for each of the adult and adolescent subgroups. Only clusters comprising more than 10 voxels are presented. All clusters showed positive correlations with performance, except for the last three clusters in the adolescent subgroup (vlPFC, VS, putamen). Talairach coordinates shown for the peak of each 3-D cluster. Probability: cluster-wise probability correlation of correlation with (A) age or (B) preference ratio. Size: number of activated voxels per 3-D cluster. 
Figure 4. Significant differences between the adult and adolescent subgroups in the correlation of performance (preference ratio) with the fidelity of neural PE representation during outcome evaluation. A and B depict significant positive (red palette) and negative (blue palette) correlations in adults and adolescents respectively. $\mathrm{C}$ additionally shows regions where the correlation coefficients were significantly different between the two groups: correlation coefficients were significantly more positive in vmPFC and cerebellum for adults and occipital cortex for adolescents and more negative in subgenual ACC and VS in adolescents. See Table 3 for full cluster descriptions.

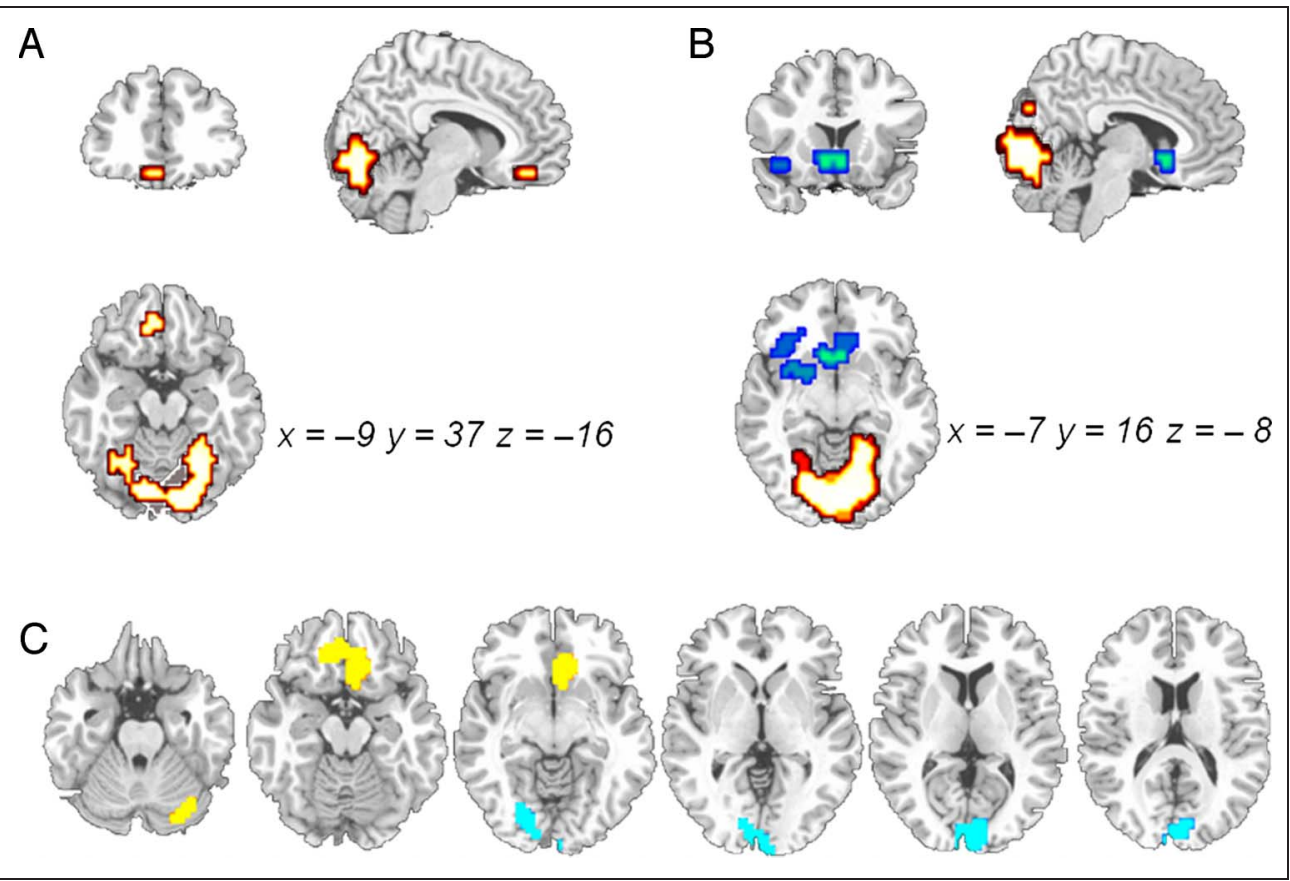

In the previous Results section, we described a qualitative difference in the association between neural PE representation and performance in adults compared with adolescents: adult individuals with higher PE representation fidelity in the vmPFC performed better, whereas adolescents with higher $\mathrm{PE}$ representation fidelity in the vlPFC, VS, putamen, and subgenual cingulate performed worse. We therefore sought to examine whether this age-specific neural PE representation pattern mapped onto different model parameters. Table 4 shows the association of PE representation in each of these clusters with each of the model parameters. The vmPFC cluster in adults was positively correlated with $\alpha^{-}$as well as outcome sensitivity, whereas the ventrolateral and subgenual PFC/ VS clusters were negatively correlated with $\alpha^{-}$.
In a final, more exploratory analysis, given prior implication of the dorsolateral frontal cortex in the task (Christakou, Brammer, et al., 2009), we found a positive correlation between the performance-predictive neural representation of decision value in dorsolateral premotor cortex (which also matured with age) and choice sensitivity, $r(35)=0.41, p<.05$, suggesting that the fidelity of value representation during decisions in this area was predictive of individual regulation of non-contingent exploration.

\section{DISCUSSION}

Using both psychological and neuroimaging measures, we investigated the maturation of reward-driven decision-
Figure 5. The fidelity of $\mathrm{PE}$ representation in the vmPFC was associated with the difference in learning rates from positive and negative PEs in adults but not in adolescents (*significant at the $p<.05$ level). Specifically, adults who showed increased PE fidelity in the vmPFC relied more on negative compared with positive predictions errors in driving future performance. BOLD signal for this analysis was extracted from a vmPFC cluster, which showed ageand performance-correlated changes in PE representation across the whole sample.

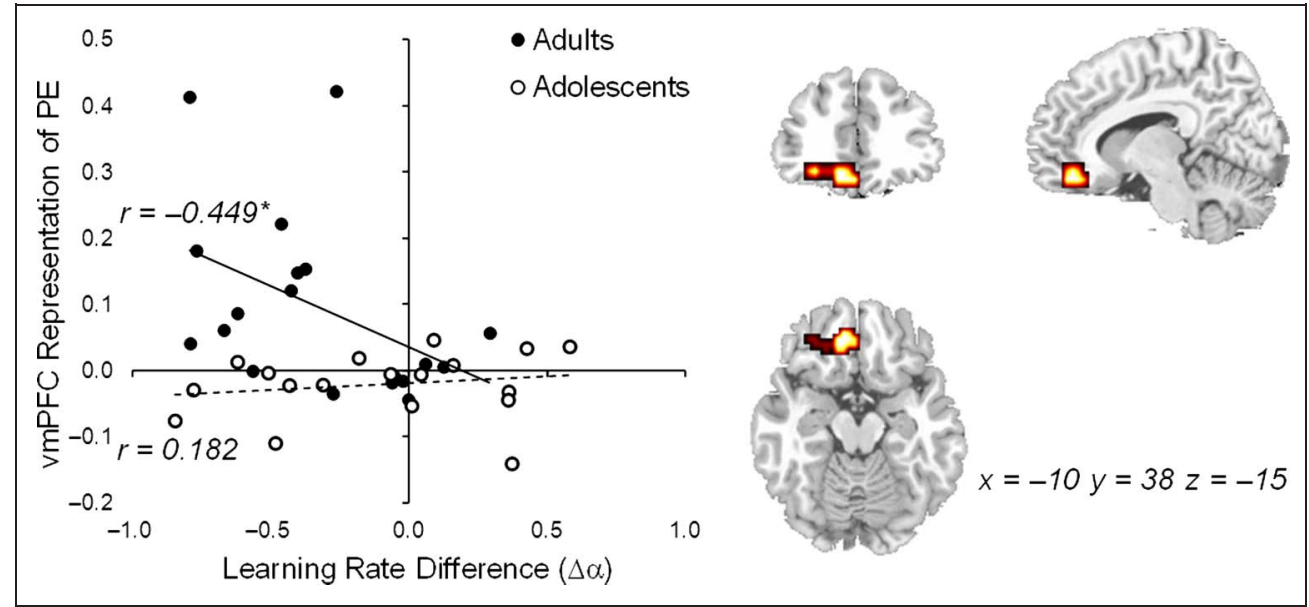


Table 4. Association between Model Parameters and Neural Representation of PE

\begin{tabular}{|c|c|c|c|c|c|}
\hline & $\begin{array}{c}\text { Outcome } \\
\text { Sensitivity (v) }\end{array}$ & $\begin{array}{c}\text { Choice } \\
\text { Sensitivity }(\omega)\end{array}$ & $\begin{array}{l}P E^{+} \text {Learning } \\
\text { Rate }(\alpha+)\end{array}$ & $\begin{array}{l}P E^{-} \text {Learning } \\
\text { Rate }\left(\alpha^{-}\right)\end{array}$ & $\begin{array}{c}\text { Learning Rate } \\
\text { Difference }(\Delta \alpha)\end{array}$ \\
\hline \multicolumn{6}{|l|}{ Adults } \\
\hline \multicolumn{6}{|l|}{ vmPFC } \\
\hline Pearson's $r$ & $.525 *$ & -.147 & -.161 & $.523 *$ & $-.542 *$ \\
\hline Sig. (2-tailed) & .021 & .548 & .511 & .022 & .017 \\
\hline \multicolumn{6}{|l|}{ Adolescents } \\
\hline \multicolumn{6}{|l|}{ vlPFC } \\
\hline Pearson's $r$ & .014 & .308 & .292 & $-.671 * *$ & $.643 * *$ \\
\hline Sig. (2-tailed) & .954 & .213 & .239 & .002 & .004 \\
\hline \multicolumn{6}{|l|}{ VS/Putamen } \\
\hline Pearson's $r$ & -.314 & .302 & .324 & -.347 & .411 \\
\hline Sig. (2-tailed) & .204 & .223 & .19 & .158 & .09 \\
\hline \multicolumn{6}{|c|}{ Subgenual ACC; VS } \\
\hline Pearson's $r$ & -.198 & .287 & .375 & -.407 & $.479 *$ \\
\hline Sig. (2-tailed) & .432 & .248 & .125 & .094 & .044 \\
\hline
\end{tabular}

Matrix of correlation between model parameters and the strength of PE representation in clusters associated with performance unique to each of the age subgroups. The adult cluster was positively correlated with preference ratio, whereas the adolescent clusters were negatively correlated with preference ratio. Only the vlPFC correlation with $\alpha^{-}$survives correction for multiple comparisons.

*Correlation is significant at the 0.05 level (two-tailed).

**Correlation is significant at the 0.01 level (two-tailed).

making between early adolescence and young adulthood with an fMRI-compatible IGT variant (Christakou, Brammer, et al., 2009). We applied a TD model of RL to parameterize individual differences and age effects on performance and brain activation, including sensitivity to the quality of decision values (V) and the impact of positive and negative PEs $\left(\alpha^{+} / \alpha^{-}\right)$.

Task performance (preference ratio for safe or advantageous compared with risky or disadvantageous decks) improved with age through adolescence stabilizing in adulthood, in line with previous behavioral studies with the IGT in this age range (Cauffman et al., 2010; Hooper, Luciana, Wahlstrom, Conklin, \& Yarger, 2008). Accordingly, sensitivity to the subjective value of the chosen option was predictive of performance and increased with age. The neural representation of decision values was shown to mature in ventromedial/ventrolateral and dorsal PFC, premotor, parahippocampal, and parietal cortex, suggesting the maturation of a network that integrates action values with memory and executive/attentional processes, as predicted by observed structural and functional maturation in this age range (Rubia, 2012; Somerville \& Casey, 2011; Casey et al., 2008; Paus, 2005).

At the same time, the neural representation of PEs during outcome evaluation matured in the vmPFC and dIPFC (in line with prior evidence, e.g., Van den Bos,
Cohen, Kahnt, \& Crone, 2012; Cohen et al., 2010), in a manner predictive of performance. We have previously shown that performance in this task depends on vmPFC and dlPFC sensitivity to worse than expected outcomes (Christakou, Brammer, et al., 2009). Here, we borrow from classical learning paradigms to examine approach and avoidance behavior (Cauffman et al., 2010) by separating the learning rates for positive and negative PEs as has been applied in other decision-making tasks (Van den Bos et al., 2012; Frank et al., 2007). We show that sensitivity to $\mathrm{PE}^{+}$and $\mathrm{PE}^{-}$had opposite effects on performance: high sensitivity to $\mathrm{PE}^{+}$was predictive of increased risky choices, whereas high sensitivity to $\mathrm{PE}^{-}$was predictive of increased safe choices. This is in line with our previous work with the same task, where performance was shown to depend on successfully tracking negative outcomes (Christakou, Brammer, et al., 2009), as well as equivalent findings with the original IGT and related tasks (Van den Bos et al., 2012; Wheeler \& Fellows, 2008; Wood, Busemeyer, Koling, Cox, \& Davis, 2005).

In our task, therefore, differential responsivity to positive and negative PEs was predictive of performance and moreover matured with age. Specifically, although adults showed significantly increased sensitivity to $\mathrm{PE}^{-}$compared with $\mathrm{PE}^{+}$, adolescents showed no difference in the rate of learning from $\mathrm{PE}^{+}$and $\mathrm{PE}^{-}$. This result expands on 
previous findings showing a difference in approach and avoidance behavior between adults and adolescents in the IGT (Cauffman et al., 2010).

Importantly, there was a negative correlation between sensitivity to $\mathrm{PE}^{+}$and performance across the sample. This indicates that reduction in individual sensitivity to better than expected outcomes (at least in similar decision-making situations) is a steady characteristic of development beyond the adolescent years and may therefore rely on experience as much as life-span developmental brain changes. By contrast, the relationship between sensitivity to $\mathrm{PE}^{-}$and performance strengthened with age only during adolescence, indicating that individual sensitivity to worse than expected outcomes may be a more sensitive marker of adolescent-specific developmental events.

This finding specifically suggests that differences between adolescent and adult behavior may be due to differences in responsivity to worse-than-expected outcomes, rather than hyper-responsiveness to better-than-expected outcomes, as has been suggested previously (Cohen et al., 2010). Likewise, this finding also contrasts the prior description of decreases in the sensitivity to negative feedback from adolescence into adulthood (Van den Bos et al., 2012). Our observation of a beneficial developmental increase in sensitivity to worse-than-expected outcomes may be restricted to situations of ambiguous contingencies, such as those in the IGT and its current variant, comprising a complex mix of positive and negative rewards. Together these observations suggest that adults and adolescents may differ in the utilization of reinforcement signals in a context-specific manner, that is, not in a matter of degree.

The wider implication of this analysis, however, is that neural and cognitive handling of outcome PEs shows a maturational profile that goes beyond reactivity to reward presentation or availability, in line with enriched models of adolescent risk-taking development (Pfeifer \& Allen, 2012; Reyna \& Farley, 2006). Accordingly, there was a qualitative difference in the way regional PE representation was associated with performance between adults and adolescents. In adults, higher PE representation fidelity in the vmPFC was associated with better performance; by contrast in adolescents, higher PE representation fidelity in the vlPFC, VS, putamen, and subgenual cingulate was associated with worse performance. This dissociation may reflect qualitative differences in the cognitive organization of decision-making in the two age groups. vmPFC represents the value of stimuli or actions as benchmarked against the motivational state and current context of the individual. By contrast, vlPFC has been suggested to specialize in credit assignment, a fundamental aspect of associative learning, which, however, lacks contextual sophistication (Rushworth, Noonan, Boorman, Walton, \& Behrens, 2011; Walton, Behrens, Buckley, Rudebeck, \& Rushworth, 2010). Analyzed in this framework, our results suggest that adolescents may lack as yet the benefit of the sophisticated contextualization of reward information provided by the vmPFC. Relying instead on vlPFC to assign outcome quality to events and on phylogenetically older limbic regions such as the subgenual ACC and VS to provide motivational information may prove counterproductive in situations where immediate values need to be discounted in favor of longer-term motivational goals.

Characteristically, the finding of maturation of PE representation in VMPFC and VS are in line with findings of increased vmPFC-VS connectivity in adults relative to adolescents in a similar context (Van den Bos et al., 2012) and during temporal discounting (Christakou et al., 2011). They likely reflect top-down control of vmPFC over striatal VS regions, which was supported in our data by the positive correlation between performance and PEmodulated vmPFC activation in adults and the negative correlation between performance and PE-modulated VS activation in adolescents.

Our findings then point to the need for contextdependent balance of cognitive and affective processes as a driver for the maturation of motivated behavior through adolescence (Van Leijenhorst et al., 2010; Crone \& Van der Molen, 2004). This complexity needs to be addressed in studies of the development of reward-driven decisionmaking.

To this end, our current design and analysis investigated the impact of noncontingent exploration tendencies (expressed as individual sensitivity to choice history or stickiness $\omega)$ to the developmental profile of performance, in light of observed increases in novelty-seeking and exploratory behaviors during adolescence (Steinberg, 2010; Spear, 2000). These behavioral characteristics are only indirectly related to reward processing but may shape the idiosyncratic decision-making strategies of adolescents, contributing to the typically observed, complex impulsive phenotype characteristic of this age group (Somerville \& Casey, 2011; Galvan, 2010; Casey et al., 2008; Reyna \& Farley, 2006; Spear, 2000). Absence of systematic noncontingent exploratory tendencies on the one hand and of noncontingent perseveration on the other (i.e., neutral choice sensitivity, as shown by adults) represents a substantial advantage in our task, which involves trial-anderror learning. In adolescent participants, however, we observed a striking pattern of increased noncontingent exploration, expressed as negative choice sensitivity, in line with evidence of nonspecific exploratory behavior characteristic of the age range (Adriani \& Laviola, 2004; Laviola, Macri, Morley-Fletcher, \& Adriani, 2003; Spear, 2000). This observation demonstrates that the power of the model to quantify the behavior of the two groups is significantly enhanced by incorporation of the choice sensitivity parameter. Importantly, choice sensitivity was the only model parameter for which the relationship with performance was explained entirely by age effects, tapping into a purely developmental aspect of behavior that would have been inaccessible to traditional behavioral analyses. 
Specifically, we demonstrate a qualitative difference in the factors that drive superior performance in adults versus adolescents. The principal feature of this dissociation was an increased, noncontingent tendency to move away from previously experienced options (negative choice sensitivity). Importantly, this tendency persisted as an adolescence-specific characteristic regardless of performance levels, demonstrating that individual levels of this sort of exploratory behavior are orthogonal to goaldirected behavior, in line with the theoretical assumptions of the model. This analysis further suggests that it is likely, although untestable in the current sample, that our model would also dissociate individuals who show naturally positive choice sensitivity, that is, a tendency to perseverate with actions or choices regardless of their adaptive value.

Neural sensitivity to the value of upcoming decisions in dorsolateral premotor cortex (BA 6) was predictive of performance and was correlated with individual levels of choice sensitivity. Although the choice sensitivity parameter is reward independent, this association suggests that to achieve neutral choice sensitivity in an environment of fluctuating rewards, one may need to effortfully regulate (possibly inhibit) individual exploratory tendencies. This is in line with evidence for the involvement of dorsolateral premotor cortex in the spatial representation of future decisions; it suggests that the influence that the memory of prior choices has on future behavior is regulated by the ability of premotor cortex to represent the value of these choices (Tanaka, Honda, \& Sadato, 2005; Tanji, 1996; Tanji \& Shima, 1994).

In the case of the current study, importantly, our results suggest that value representation during decisions mediates improvements in performance with maturing age by reducing exploratory behavior (specifically by "neutralizing" choice sensitivity from negative to zero) and not by reducing the hedonic impact of reward on behavior. This is an important observation, further emphasizing the complexity of the maturational profile of decision-making. It further provides a new avenue of investigation related to adolescent vulnerability to pathological impulse control, such as in ADHD (Sonuga-Barke, 2005; Sagvolden \& Sergeant, 1998), as well as conditions in the opposite side of the spectrum, such as autism, where behavioral rigidity is instead a key feature (Baron-Cohen \& Belmonte, 2005). We note, however, that further research will need to address limitations of the current study, for example, by including both males and females to consider the differences between the sexes and taking into account the effects of puberty. Furthermore, larger sample sizes will be required to clarify the effects of putatively identifiable phases of adolescent development.

In summary, we used a TD RL model to explain maturation in performance of our IGT variant. We demonstrate the multidimensional nature of decision-making differences between adolescents and adults and disentangle some of the psychological and neural substrates of matur- ing performance. Accordingly, we propose the expansion of current models of adolescent development of rewarddriven processes (Pfeifer \& Allen, 2012) to include characteristics beyond the assumed hypersensitivity to reward presentation, such as individual differences in exploration and perseveration traits. This strategy has the potential to describe age-typical behaviors in increasingly complex, real-world decision-making situations. This ability, in turn, will help understand in more detail the neuropsychopathology of developmental conditions, such as affective disorders, ADHD, and autism.

\section{Acknowledgments}

The study and A. C. were supported by a Medical Research Council grant (GO300155) to K. R., a Royal Society International Travel Grant for Collaboration (TG091648) to A. C., a Human Frontier Science Program grant to A. C., and a University of Reading Research Travel Grant to A. C. Y. N. was supported by a Sloan Research Fellowship. S. J. G. was supported by an NSF Graduate Research Fellowship. A. S. was supported by the NIHR Biomedical Research Centre for Mental Health at South London and Maudsley NHS Foundation Trust and King's College London Institute of Psychiatry. The authors would like to thank Jeff Dalton for programming, Jason Stretton for assistance with data collection, Dr. Vincent Giampietro for assistance with data analysis, the staff at the Centre for Neuroimaging Sciences for their help and expert advice, and the staff at the Princeton Neuroscience Institute for facilitating our collaboration.

Reprint requests should be sent to Dr. Anastasia Christakou, Centre for Integrative Neuroscience and Neurodynamics, School of Psychology and Clinical Language Sciences, University of Reading, Whiteknights, Reading RG6 6AL, United Kingdom, or via e-mail: anastasia.christakou@reading.ac.uk.

\section{REFERENCES}

Adriani, W., \& Laviola, G. (2004). Windows of vulnerability to psychopathology and therapeutic strategy in the adolescent rodent model. Behavioural Pharmacology, 15, 341-352.

Baron-Cohen, S., \& Belmonte, M. K. (2005). Autism: A window onto the development of the social and the analytic brain. Annual Review of Neuroscience, 28, 109-126.

Bechara, A., Damasio, A. R., Damasio, H., \& Anderson, S. W. (1994). Insensitivity to future consequences following damage to human prefrontal cortex. Cognition, 50, 7-15.

Bjork, J. M., Knutson, B., Fong, G. W., Caggiano, D. M., Bennett, S. M., \& Hommer, D. W. (2004). Incentive-elicited brain activation in adolescents: Similarities and differences from young adults. The Journal of Neuroscience: The Official Journal of the Society for Neuroscience, 24, 1793-1802.

Bjork, J. M., Smith, A. R., Chen, G., \& Hommer, D. W. (2010). Adolescents, adults and rewards: Comparing motivational neurocircuitry recruitment using fMRI. PloS One, 5, e11440.

Blair, R. J., Colledge, E., \& Mitchell, D. G. (2001). Somatic markers and response reversal: Is there orbitofrontal cortex dysfunction in boys with psychopathic tendencies? Journal of Abnormal Child Psychology, 29, 499-511.

Bullmore, E., Brammer, M., Rabe-Hesketh, S., Curtis, V., Morris, R., Williams, S., et al. (1999). Methods for diagnosis and treatment of stimulus-correlated motion in generic brain activation studies using fMRI. Human Brain Mapping, 7, 38-48. 
Bullmore, E., Long, C., Suckling, J., Fadili, J., Calvert, G., Zelaya, F., et al. (2001). Colored noise and computational inference in neurophysiological (fMRI) time series analysis: Resampling methods in time and wavelet domains. Human Brain Mapping, 12, 61-78.

Casey, B. J., Getz, S., \& Galvan, A. (2008). The adolescent brain. Developmental Review, 28, 62-77.

Cauffman, E., Shulman, E. P., Steinberg, L., Claus, E., Banich, M. T., Graham, S., et al. (2010). Age differences in affective decision making as indexed by performance on the Iowa gambling task. Developmental Psychology, 46, 193-207.

Christakou, A., Brammer, M., Giampietro, V., \& Rubia, K. (2009). Right ventromedial and dorsolateral prefrontal cortices mediate adaptive decisions under ambiguity by integrating choice utility and outcome evaluation. Journal of Neuroscience, 29, 11020-11028.

Christakou, A., Brammer, M., \& Rubia, K. (2011). Maturation of limbic corticostriatal activation and connectivity associated with developmental changes in temporal discounting. Neuroimage, 54, 1344-1354.

Christakou, A., Halari, R., Smith, A. B., Ifkovits, E., Brammer, M., \& Rubia, K. (2009). Sex-dependent age modulation of frontostriatal and temporo-parietal activation during cognitive control. Neuroimage, 48, 223-236.

Cohen, J. R., Asarnow, R. F., Sabb, F. W., Bilder, R. M., Bookheimer, S. Y., Knowlton, B. J., et al. (2010). A unique adolescent response to reward prediction errors. Nature Neuroscience, 13, 669-671.

Crone, E. A., \& Van der Molen, M. W. (2004). Developmental changes in real life decision making: Performance on a gambling task previously shown to depend on the ventromedial prefrontal cortex. Developmental Neuropsychology, 25, 251-279.

Crone, E. A., \& Van der Molen, M. W. (2007). Development of decision making in school-aged children and adolescents: Evidence from heart rate and skin conductance analysis. Child Development, 78, 1288-1301.

Ernst, M., \& Fudge, J. L. (2009). A developmental neurobiological model of motivated behavior: Anatomy, connectivity and ontogeny of the triadic nodes. Neuroscience and Biobehavioral Reviews, 33, 367-382.

Eshel, N., Nelson, E. E., Blair, R. J., Pine, D. S., \& Ernst, M. (2007). Neural substrates of choice selection in adults and adolescents: Development of the ventrolateral prefrontal and anterior cingulate cortices. Neuropsychologia, 45, 1270-1279.

Frank, M. J., Moustafa, A. A., Haughey, H. M., Curran, T., \& Hutchison, K. E. (2007). Genetic triple dissociation reveals multiple roles for dopamine in reinforcement learning. Proceedings of the National Academy of Sciences, U.S.A., 104, 16311-16316.

Galvan, A. (2010). Adolescent development of the reward system. Frontiers in Human Neuroscience, 4, 6.

Galvan, A., Hare, T. A., Parra, C. E., Penn, J., Voss, H., Glover, G., et al. (2006). Earlier development of the accumbens relative to orbitofrontal cortex might underlie risk-taking behavior in adolescents. The Journal of Neuroscience: The Official Journal of the Society for Neuroscience, 26, 6885-6892.

Gershman, S. J., Pesaran, B., \& Daw, N. D. (2009). Human reinforcement learning subdivides structured action spaces by learning effector-specific values. The Journal of Neuroscience, 29, 13524-13531.

Giedd, J. N., Blumenthal, J., Jeffries, N. O., Castellanos, F. X., Liu, H., Zijdenbos, A., et al. (1999). Brain development during childhood and adolescence: A longitudinal MRI study. Nature Neuroscience, 2, 861-863.

Hooper, C. J., Luciana, M., Conklin, H. M., \& Yarger, R. S. (2004). Adolescents' performance on the Iowa gambling task: Implications for the development of decision making and ventromedial prefrontal cortex. Developmental Psychology, 40, 1148-1158.

Hooper, C., Luciana, M., Wahlstrom, D., Conklin, H., \& Yarger, R. (2008). Personality correlates of Iowa gambling task performance in healthy adolescents. Personality and Individual Differences, 44, 598-609.

Huizenga, H. M., Crone, E. A., \& Jansen, B. J. (2007). Decision-making in healthy children, adolescents and adults explained by the use of increasingly complex proportional reasoning rules. Developmental Science, 10, 814-825.

Jeffreys, H. (1961). Theory of probability. Oxford: Oxford University Press.

Kass, R. E., \& Raftery, A. E. (1995). Bayes factors. Journal of the American Statistical Association, 90, 773-795.

Lau, B., \& Glimcher, P. W. (2005). Dynamic response-by-response models of matching behavior in rhesus monkeys. Journal of the Experimental Analysis of Behavior, 84, 555-579.

Laviola, G., Macri, S., Morley-Fletcher, S., \& Adriani, W. (2003). Risk-taking behavior in adolescent mice: Psychobiological determinants and early epigenetic influence. Neuroscience \& Biobehavioral Reviews, 27, 19-31.

Lawrence, N. S., Jollant, F., O’Daly, O., Zelaya, F., \& Phillips, M. L. (2009). Distinct roles of prefrontal cortical subregions in the Iowa gambling task. Cerebral Cortex (New York, NY: 1991), 19, 1134-1143.

Niv, Y., Edlund, J. A., Dayan, P., \& O’Doherty, J. P. (2012). Neural prediction errors reveal a risk-sensitive reinforcement-learning process in the human brain. Journal of Neuroscience, 32, 551-562.

Oldfield, R. C. (1971). The assessment and analysis of handedness: The Edinburgh inventory. Neuropsychologia, 9, 97-113.

Paus, T. (2005). Mapping brain maturation and cognitive development during adolescence. Trends in Cognitive Sciences, 9, 60-68.

Pfeifer, J. H., \& Allen, N. B. (2012). Arrested development? Reconsidering dual-systems models of brain function in adolescence and disorders. Trends in Cognitive Sciences, 16, 322-329.

Reyna, V. F., \& Farley, F. (2006). Risk and rationality in adolescent decision making: Implications for theory, practice, and public policy. Psychological Science in the Public Interest, 7, 1-44.

Rubia, K. (2012). Functional brain imaging across development. European Child \& Adolescent Psychiatry. Doi:10.1007/ s00787-012-0291-8.

Rubia, K., Hyde, Z., Halari, R., Giampietro, V., \& Smith, A. (2010). Effects of age and sex on developmental neural networks of visual-spatial attention allocation. Neuroimage, 51, 817-827.

Rubia, K., Overmeyer, S., Taylor, E., Brammer, M., Williams, S., Simmons, A., et al. (2000). Functional frontalisation with age: Mapping neurodevelopmental trajectories with fMRI. Neuroscience and Biobehavioral Reviews, 24, 13-19.

Rubia, K., Smith, A. B., Taylor, E., \& Brammer, M. (2007). Linear age-correlated functional development of right inferior fronto-striato-cerebellar networks during response inhibition and anterior cingulate during error-related processes. Human Brain Mapping, 28, 1163-1177.

Rubia, K., Smith, A. B., Woolley, J., Nosarti, C., Heyman, I., Taylor, E., et al. (2006). Progressive increase of frontostriatal brain activation from childhood to adulthood during event-related tasks of cognitive control. Human Brain Mapping, 27, 973-993.

Rushworth, M. F. S., Noonan, M. P., Boorman, E. D., Walton, M. E., \& Behrens, T. E. (2011). Frontal cortex and reward-guided learning and decision-making. Neuron, 70, 1054-1069. 
Sagvolden, T., \& Sergeant, J. A. (1998). Attention deficit/ hyperactivity disorder-From brain dysfunctions to behaviour. Behavioural Brain Research, 94, 1-10.

Simmons, A., Moore, E., \& Williams, S. C. R. (1999). Quality control for functional magnetic resonance imaging using automated data analysis and Shewhart charting. Magnetic Resonance in Medicine, 41, 1274-1278.

Simon, D. A., \& Daw, N. D. (2011). Neural correlates of forward planning in a spatial decision task in humans. Journal of Neuroscience, 31, 5526-5539.

Smith, A. B., Halari, R., Giampetro, V., Brammer, M., \& Rubia, K. (2011). Developmental effects of reward on sustained attention networks. Neuroimage, 56, 1693-1704.

Smith, D. G., Xiao, L., \& Bechara, A. (2012). Decision making in children and adolescents: Impaired Iowa gambling task performance in early adolescence. Developmental Psychology, 48, 1180-1187.

Somerville, L. H., \& Casey, B. J. (2011). Developmental neurobiology of cogntive control and motivational systems. Current Opinion in Neurobiology, 20, 236-241.

Sonuga-Barke, E. J. S. (2005). Causal models of attention-deficit/ hyperactivity disorder: From common simple deficits to multiple developmental pathways. Biological Psychiatry, 57, 1231-1238.

Sowell, E. R., Thompson, P. M., Holmes, C. J., Batth, R., Jernigan, T. L., \& Toga, A. W. (1999). Localizing age-related changes in brain structure between childhood and adolescence using statistical parametric mapping. Neuroimage, 9, 587-597.

Spear, L. P. (2000). The adolescent brain and age-related behavioral manifestations. Neuroscience and Biobehavioral Reviews, 24, 417-463.

Steinberg, L. (2010). A dual systems model of adolescent risk-taking. Developmental Psychobiology, 52, 216-224.

Stephan, K. E., Penny, W. D., Daunizeau, J., Moran, R. J., \& Friston, K. J. (2009). Bayesian model selection for group studies. Neuroimage, 46, 1004-1017.

Sutton, R. S., \& Barto, A. G. (1998). Reinforcement learning: An introduction. Cambridge, MA: MIT Press.

Tanaka, S., Honda, M., \& Sadato, N. (2005). Modality-specific cognitive function of medial and lateral human Brodmann area 6. The Journal of Neuroscience: The Official Journal of the Society for Neuroscience, 25, 496-501.

Tanji, J. (1996). New concepts of the supplementary motor area. Current Opinion in Neurobiology, 6, 782-787.
Tanji, J., \& Shima, K. (1994). Role for supplementary motor area cells in planning several movements ahead. Nature, 371, 413-416.

Teicher, M. H., Andersen, S. L., \& Hostetter, J. C. (1995). Evidence for dopamine receptor pruning between adolescence and adulthood in striatum but not nucleus accumbens. Brain Research Developmental Brain Research, 89, 167-172.

Thirion, B., Pinel, P., Mériaux, S., Roche, A., Dehaene, S. \& Poline, J.-B. (2007). Analysis of a large fMRI cohort: Statistical and methodological issues for group analyses. Neuroimage, 35, 105-120.

Tranel, D., Damasio, H., Denburg, N. L., \& Bechara, A. (2005). Does gender play a role in functional asymmetry of ventromedial prefrontal cortex? Brain: A Journal of Neurology, 128, 2872-2881.

Van den Bos, W., Cohen, M. X., Kahnt, T., \& Crone, E. A. (2012). Striatum-medial prefrontal cortex connectivity predicts developmental changes in reinforcement learning. Cerebral Cortex, 22, 1247-1255.

Van Duijvenvoorde, A. C. K., Jansen, B. R. J., Visser, I., \& Huizenga, H. M. (2010). Affective and cognitive decision-making in adolescents. Developmental Neuropsychology, 35, 539-554.

Van Leijenhorst, L., Moor, B. G., Op de Macks, Z. A., Rombouts, S. A. R. B., Westenberg, P. M., \& Crone, E. A. (2010). Adolescent risky decision-making: Neurocognitive development of reward and control regions. Neuroimage, 51, 345-355.

Walton, M. E., Behrens, T. E. J., Buckley, M. J., Rudebeck, P. H., \& Rushworth, M. F. S. (2010). Separable learning systems in the macaque brain and the role of orbitofrontal cortex in contingent learning. Neuron, 65, 927-939.

Wheeler, E. Z., \& Fellows, L. K. (2008). The human ventromedial frontal lobe is critical for learning from negative feedback. Brain: A Journal of Neurology, 131, 1323-1331.

Wills, T. A., Vaccaro, D., \& McNamara, G. (1994). Novelty seeking, risk taking, and related constructs as predictors of adolescent substance use: An application of Cloninger's theory. Journal of Substance Abuse, 6, 1-20.

Wood, S., Busemeyer, J., Koling, A., Cox, C. R., \& Davis, H. (2005). Older adults as adaptive decision makers: Evidence from the Iowa gambling task. Psychology and Aging, 20, 220-225. 